\title{
NOUVELLE
}

\section{Apport des modèles murins dans l'autisme syndromique et non syndromique}

Une physiopathologie commune?

Stéphane J. Baudouin
Research group of Peter Scheiffele,

Mechanisms in the formation of neuronal networks,

Biozentrum of the University of Basel, Klingelbergstrasse 50-70, 4056 Basel, Suisse.

stephane.baudouin@unibas.ch
De la génétique à la physiopathologie des troubles du spectre autistique L'autisme est une maladie neurodéveloppementale initialement caractérisée par Léo Kanner en 1946. Les symptômes centraux, définis par l'Association américaine de psychiatrie dans le Diagnostic and statistical manual of mental disorders IV (DSM-IV) et qui font l'objet d'un consensus international sont « un développement nettement anormal ou déficient de l'interaction sociale et de la communication, et un répertoire considérablement restreint d'activités et d'intérêts ». Chez un grand nombre de patients, à ces symptômes centraux de l'autisme s'associent d'autres caractéristiques cliniques, comme des déficiences intellectuelles, l'épilepsie, l'hyperactivité. D'autres ont des formes d'autisme appelées syndromiques où l'autisme ne constitue qu'une partie du diagnostic : par exemple, les syndromes de I'X-fragile [1], de Rett [2] ou la sclérose tubéreuse de Bourneville [3]. On définit aujourd'hui deux catégories d'autisme: l'autisme syndromique et l'autisme non syndromique, toutes deux regroupées sous le terme générique de troubles du spectre autistique (Figure 1). La redéfinition de l'autisme s'est accompagnée d'une modification et d'une harmonisation des critères diagnostiques conduisant à une augmentation de la prévalence de l'autisme, qui est passée d'environ 4/10 000 il y a 20 ans à environ $1 / 100$ aujourd'hui. Le diagnostic peut être posé dès l'âge de deux ans de manière fiable, l'âge moyen du diagnostic étant d'un peu plus de trois ans. La pathologie touche quatre fois plus de garçons que de filles. Le traitement de l'autisme est à l'heure actuelle entièrement basé sur des thérapies comportementales; il n'existe pas de traitement médicamenteux.

À la fin des années 1980, une première série d'études génétiques démontra clairement le caractère hautement héréditaire de l'autisme avec un taux d'environ $90 \%$ (contre $80 \%$ pour la schizophrénie et $40 \%$ pour la maladie de Parkinson). Des analyses systématiques de l'ensemble du génome sur de larges cohortes de patients ont alors mis en évidence de nombreuses mutations pouvant être à l'origine de la pathologie [4]. Le premier constat des études génétiques menées jusqu'à aujourd'hui est que l'autisme n'est pas monogénique, mais plus probablement dû à l'apparition simultanée, chez un même individu, de plusieurs mutations sur plusieurs gènes. Ces mutations peuvent alors avoir des effets additifs ou combinatoires. Le second apport des analyses génétiques fut l'identification de fonctions biologiques potentiellement altérées dans l'autisme. Un grand nombre de mutations ont, par exemple, été identifiées sur des gènes codant pour des protéines ayant des fonctions synaptiques [5]. La mise en évidence de gènes impliqués dans la survenue des troubles du spectre autistique a alors permis de concevoir des modèles de souris de la maladie dans le but d'étudier la pathologie sur le plan moléculaire, cellulaire et physiologique. La démarche est simple : elle consiste à introduire, par transgenèse, la mutation chez la souris. La validation du modèle passe par une analyse comportementale des souris centrée sur la recherche de l'équivalent des symptômes centraux chez l'humain : des défauts de communication, de sociabilité et l'apparition de comportements répétitifs [6] (Figure 2).

\section{La modélisation des syndromes}

\section{associés à l'autisme}

Les plus grandes réussites de cette approche concernent les formes syndromiques monogéniques de l'autisme, et en particulier le syndrome de l'X-fragile. Le syndrome de l' $X$-fragile est dû à une mutation du gène fragile $X$ mental retardation 1 (FMRI), codant pour FMRP, une protéine régulatrice de la traduction [1]. Cette mutation entraîne sur le plan clinique un retard mental plus ou moins sévère, une macro-orchidie, une mâchoire proéminente, de grandes oreilles, un déficit de l'attention et de la concentration et, dans $30 \%$ des cas, un autisme. La mutation du gène correspondant chez la souris induit l'apparition de symptômes similaires à ceux qui sont observés chez I'humain. Parmi les caractéristiques physiopathologiques de ces souris, ont été mis en évidence : I'hyperactivité d'un sous-type particulier de récepteurs du glutamate, les récepteurs métabotropiques (mGlu) de type 1, une altération de la morphologie synaptique et un défaut 


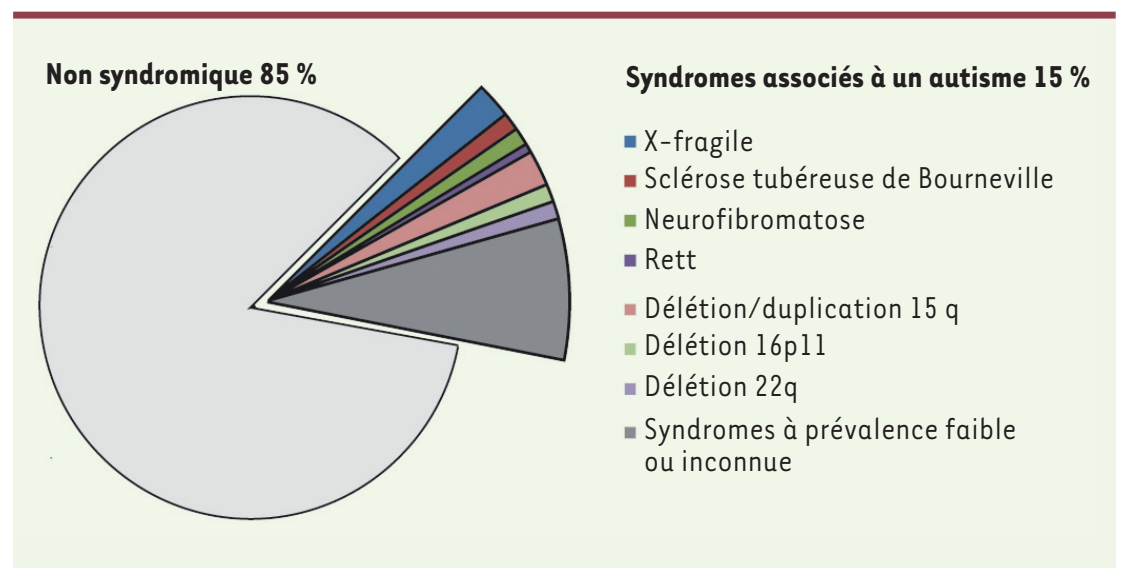

Figure 1. Prévalence des formes syndromiques et non syndromiques dans les troubles du spectre autistique. Les pathologies auxquelles est associé un diagnostic d'autisme sont regroupées sous le terme générique de trouble du spectre autistique. Les troubles du spectre autistique ont une origine génétique clairement identifiée pour les autismes d'origine syndromique (il s'agit soit d'une mutation d'un gène, soit de la délétion ou duplication d'une partie d'un chromosome) alors qu'elle est inconnue dans la majorité des cas d'autisme non syndromique [15].

de régulation de la plasticité synaptique appelée dépression à long terme (un mécanisme cellulaire à la base des processus de mémorisation). Ces résultats ont abouti à la «théorie des récepteurs mGlu » qui postule que le dysfonctionnement des récepteurs mGlu de type 1 est la cause principale de l'apparition des symptômes autistiques, définissant ainsi les récepteurs mGlu de type 1 comme une cible thérapeutique [7]. L'utilisation de modulateurs négatifs des récepteurs mGlu de type 1 s'avère ainsi aujourd'hui une stratégie thérapeutique prometteuse. Ce type de traitement chez la souris adulte a ainsi permis la correction presque complète des altérations cellulaires et comportementales [8]. II est actuellement en cours de validation chez l'humain (http://clinicaltrials.gov).

\section{Vers une généralisation des stratégies} thérapeutiques dans les troubles du spectre autistique

Une des questions soulevées par les travaux sur le syndrome de l'X-fragile est de savoir si la thérapie corrige le syndrome (et donc les symptômes centraux de l'autisme qui lui sont associés), ou s'il est possible de corriger directement les symptômes centraux de l'autisme en ciblant les récepteurs mGlu de type 1 . Au-delà, se pose la question de l'application de cette stratégie chez l'adulte: peut-elle fonctionner à un stade avancé de la maturation cérébrale ? L'approche que nous avons utilisée a été de choisir un gène impliqué dans l'autisme, mais dont la mutation n'est associée à aucun signe clinique supplémentaire, donc un autisme non syndromique. Le gène NLGN3 est porté par le chromosome $X$ et code pour la protéine d'adhésion synaptique, la neuroligine 3. NLGN3 appartient à la famille des neuroligines qui compte cinq membres chez l'humain; les neuroligines 1 et 4 sont aussi impliquées dans l'autisme. Les mutations sur NLGN3 sont rares, mais lorsqu'elles existent, la prévalence d'un trouble autistique est de $100 \%$ [9]. Des travaux précédents avaient montré que les souris porteuses des mutations de NGLN3 liées à la survenue d'un autisme présentent des altérations comportementales semblables à celles qui caractérisent l'autisme humain [10]. Sur le plan physiopathologique, peu de données étaient disponibles. Nous avons alors analysé de manière systématique les altérations synaptiques dans certaines synapses du cervelet, une région du cerveau impliquée dans le contrôle du mouvement et des émotions, et dans l'apparition des symptômes associés à l'autisme [11]. L'originalité de notre approche réside dans la stratégie génétique utilisée. Nous avons généré une souris transgénique dans laquelle il est possible de contrôler la réexpression de la neuroligine 3 dans des sous-types cellulaires spécifiques et à des stades précis du développement [12]. Dans un premier temps, nous avons identifié la localisation synaptique précise de la neuroligine 3 dans les cellules de Purkinje, cellules qui ont un rôle majeur dans le cervelet car elles constituent l'unique voie de sortie de l'information traitée par ce dernier. Nous avons montré que la neuroligine 3 est présente uniquement dans un sous-type de synapses excitatrices formées par des fibres appelées parallèles. Lorsque la neuroligine 3 est absente, ces synapses expriment un niveau anormalement élevé du récepteur mGluRl (un récepteur mGlu de type 1), corrélé, au niveau fonctionnel, à une disparition de la dépression à long terme et à un déficit de coordination motrice. Sur le plan morphologique, l'absence de neuroligine 3 a pour conséquence la formation de synapses excitatrices aberrantes par des fibres appelées grimpantes (où neuroligine 3 n'est pas localisée). Nous avons ensuite analysé, en deux temps, s'il était possible d'annuler ces altérations par une réexpression de la protéine. Nous avons ainsi d'abord démontré que la réexpression constitutive et spécifique de neuroligine 3 dans les cellules de Purkinje est suffisante pour corriger l'ensemble des altérations physiopathologiques que nous avions observées. Ensuite, nous avons mis en évidence qu'une réexpression, à des stades adultes du développement cérébral, était suffisante pour restaurer des niveaux normaux de mGluRl et pour éliminer les connexions synaptiques aberrantes. Ces résultats mettent en évidence la possibilité de corriger des aberrations synaptiques chez l'adulte, qu'elles soient fonctionnelles ou morphologiques, dans un modèle murin d'autisme non 


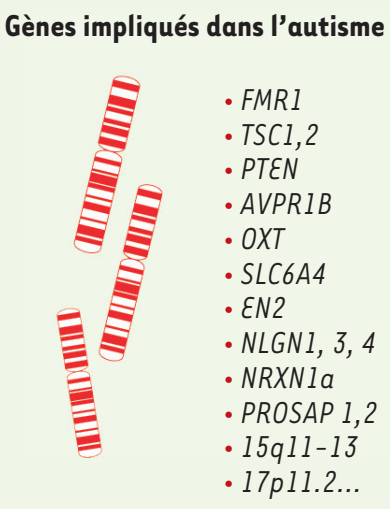

Tests comportementaux

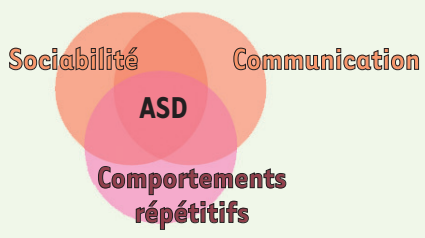

Altération de la morphologie synaptique

Altération de la balance synaptique : compétition synaptique, balance inhibition/excitation

Altération de la plasticité synaptique

Altération de la signalisation des récepteurs mGlu de type 1

- Hyperfonction dans FMR $1^{K 0}$ et NLGN3 ${ }^{K O}$

- Hypofonction dans PROSAP ${ }^{\mathrm{KO}}$ et $T S C 1,2^{\mathrm{KO}}$

Figure 2. Analyse de la physiopathologie des troubles du spectre autistique. La diversité des formes d'autisme chez l'humain rend la compréhension physiopathologique de ces troubles très complexe. Une des voies de simplification possible est la modélisation chez la souris. La démarche scientifique d'établissement d'un modèle murin se fait en deux temps: (1) introduire génétiquement une mutation chez l'animal, et (2) valider le modèle sur un plan comportemental. L'analyse transversale des modèles entre eux permet de mettre en évidence des caractéristiques physiopathologiques communes, afin de définir des stratégies thérapeutiques potentielles. ASD : autism spectrum disorders; PROSAP1 : proline-rich synapse-associated protein-1; $\mathrm{TSC} 1$ : tuberous sclerosis protein 1 .

syndromique. Comme c'était le cas pour le syndrome de l'X-fragile, nous avons observé un défaut de plasticité synaptique associé à une hyperactivation des récepteurs mGlu de type 1 . Ceci permet d'identifier une physiopathologie commune aux formes syndromiques et non syndromiques de l'autisme et, ainsi, de définir une stratégie thérapeutique potentielle commune, via la modulation négative de la fonction des récepteurs mGlu de type 1 .

\section{Conclusion et perspectives}

Ce domaine de recherche est encore jeune, mais extrêmement dynamique, en témoigne l'apparition quasi constante de nouveaux modèles animaux de cette pathologie [6]. La comparaison de ces modèles entre eux, sur le plan physiopathologique, devrait permettre de continuer à mieux définir la maladie et de découvrir de nouvelles stratégies thérapeutiques (Figure 2). La stratégie visant à cibler les récepteurs mGlu de type 1 est un bon exemple, même si certaines questions, quant au niveau de contrôle des récepteurs, restent en suspens [13, 14]. Pour finir, il est important de noter que ces résultats trouvent un écho chez I'humain, puisque les premiers essais thérapeutiques basés sur l'utilisation de modulateurs négatifs de la fonction des récepteurs mGlu de type 1 sont en cours. Ils sont une source évidente d'espoir pour les patients, mais leurs résultats sont attendus par les chercheurs, car ils devraient ajouter une dimension supplémentaire à la valeur de la recherche sur les modèles animaux. $\diamond$

Mouse models of autism: a common basis for syndromic and non syndromic autisms?

\section{LIENS D'INTÉRÊT}

L'auteur déclare n'avoir aucun lien d'intérêt concernant les données publiées dans cet article.

\section{RéFÉRENCES}

1. Davidovic L, Tremblay S, Gravel M, et al. Le syndrome de l'X fragile : une protéine absente et 1001 ARNm déboussolés. Med Sci (Paris) 2006 ; 22 : 41-6.

2. Roux JC, Villard L. Traitement pharmacologique dans le syndrome de Rett : des résultats prometteurs chez la souris. Med Sci (Paris) 2007 ; 23 : 805-7.

3. Feliciano D, Bordey $A$. Un modèle murin de sclérose tubéreuse de Bourneville pour comprendre le processus d'épilepsie associé aux tubers corticaux. Med Sci (Paris) 2011 ; $27: 328-30$.

4. Jamain $S$, Betancur C, Giros B, et al. La génétique de l'autisme. Med Sci (Paris) 2003 ; 19 : 181-90.

5. Zoghbi Hy. Postnatal neurodevelopmental disorders: meeting at the synapse? Science $2003 ; 302$ : 826-30.

6. Silverman JL, Yang M, Lord C, Crawley JN. Behavioural phenotyping assays for mouse models of autism. Nat Rev Neurosci 2010 ; 11 : 490-502.
7. Bear MF, Huber KM, Warren ST. The mGluR theory of fragile $X$ mental retardation. Trends Neurosci 2004 ; 27 ; 370-7.

8. Michalon A, Sidorov M, Ballard TM, et al. Chronic pharmacological mGlu 5 inhibition corrects fragile $X$ in adult mice. Neuron $2012 ; 74: 49-56$.

9. Jamain S, Quach H, Betancur C, et al. Mutations of the $X$-linked genes encoding neuroligins NLGN3 and NLGN4 are associated with autism. Nat Genet $2003 ; 34: 27-9$.

10. Radyushkin K, Hammerschmidt K, Boretius S, et al. Neuroligin-3-deficient mice: model of a monogenic heritable form of autism with an olfactory deficit. Genes Brain Behav $2009 ; 8$ : 416-25.

11. Tsai PT, Hull C, Chu Y, et al. Autistic-like behaviour and cerebellar dysfunction in Purkinje cell Tscl mutant mice. Nature $2012 ; 488$ : 647-51.

12. Baudouin SJ, Gaudias J, Gerharz S, et al. Shared synaptic pathophysiology in syndromic and nonsyndromic rodent models of autism. Science 2012 ; 338 : 128-32.

13. Auerbach BD, Osterweil EK, Bear MF. Mutations causing syndromic autism define an axis of synaptic pathophysiology. Nature $2011 ; 480: 63-8$.

14. Won H, Lee HR, Gee Hy, et al. Autistic-like social behaviour in Shank2-mutant mice improved by restoring NMDA receptor function. Nature 2012 ; 486 : 261-5.

15. Abrahams BS, Geschwind DH. Advances in autism genetics: on the threshold of a new neurobiology. Nat Rev Genet 2008 ; 9 : 341-55. 